\title{
Interaction of a Screw Dislocation with Interface and Wedge-Shaped Cracks in One-Dimensional Hexagonal Piezoelectric Quasicrystals Bimaterial
}

\author{
Lian he Li $(\mathbb{D})$ and Yue Zhao $\mathbb{D}$ \\ College of Mathematics Science, Inner Mongolia Normal University, Huhhot 010022, China \\ Correspondence should be addressed to Lian he Li; nmglilianhe@163.com
}

Received 19 December 2018; Accepted 25 March 2019; Published 17 April 2019

Academic Editor: Xiao-Qiao He

Copyright (C) 2019 Lian he Li and Yue Zhao. This is an open access article distributed under the Creative Commons Attribution License, which permits unrestricted use, distribution, and reproduction in any medium, provided the original work is properly cited.

\begin{abstract}
Interaction of a screw dislocation with wedge-shaped cracks in one-dimensional hexagonal piezoelectric quasicrystals bimaterial is considered. The general solutions of the elastic and electric fields are derived by complex variable method. Then the analytical expressions for the phonon stresses, phason stresses, and electric displacements are given. The stresses and electric displacement intensity factors of the cracks are also calculated, as well as the force on dislocation. The effects of the coupling constants, the geometrical parameters of cracks, and the dislocation location on stresses intensity factors and image force are shown graphically. The distribution characteristics with regard to the phonon stresses, phason stresses, and electric displacements are discussed in detail. The solutions of several special cases are obtained as the results of the present conclusion.
\end{abstract}

\section{Introduction}

As a new structure of solid in nature, quasicrystals (QCs) differ from ordinary crystals and noncrystals. QCs have many excellent properties, such as high strength, high wear resistance, and low heat-transfer, which have great development trends and been applied extensively in the fields of technology and engineering. Since the discovery of QCs [1], generalized elasticity theory of QCs has been established $[2,3]$. Using the mathematical theory of elasticity of QCs, Fan et al. [4,5] studied the moving screw dislocation and straight dislocation in one-dimensional (1D) hexagonal QCs. Enrico et al. [6] studied the linear crack problem in ten symmetric twodimensional quasicrystals by using the Stroh method. Gao et al. [7] solved the fracture mechanics problem of cubic quasicrystals with an elliptical hole or a crack. Wang et al. [8] coped with the problem involving the interaction between a screw dislocation and a semi-infinite crack in decagonal QCs. Liu et al. [9] studied the interaction of dislocations with cracks in one-dimensional hexagonal QCs based on the analytic function theory. Li et al. [10] investigated the interaction of a screw dislocation with an elliptical hole in icosahedral quasicrystals.
Piezoelectricity is an important physical property of QCs. The piezoelectric QCs [11-14] have been investigated to a certain degree. It is well known that the interaction among defects is of considerable importance in understanding the fracture mechanical behavior of materials. Few results have been reported with regard to the interaction problem of defects in piezoelectric QCs.

Wedge-shaped crack is an important geometric form of many composites and structures. The discontinuity of material properties or the abrupt change in geometry makes the stresses field at the top of wedge singular under certain conditions, which means that the wedge tip is prone to cracking. Therefore, it is of great significance for understanding the properties to study a screw dislocation interacting with interface and interfacial wedge-shaped cracks in piezoelectric quasicrystal bimaterial. The general expressions of the complex potentials are obtained and fracture parameters such as intensity factors and image force are given in closed-form. Three special cases, i.e., the screw dislocation interacting, respectively, with semi-infinite, finite, and wedge-shaped cracks in $1 \mathrm{D}$ hexagonal piezoelectric QCs bimaterial is also considered. 


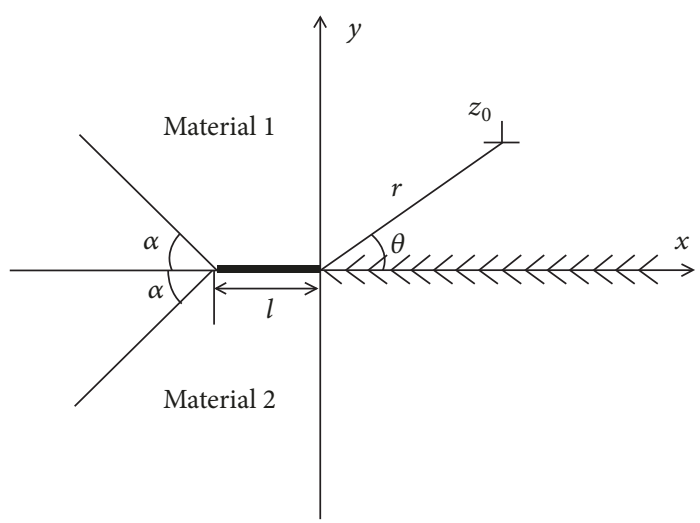

FIGURE 1: A screw dislocation interaction with wedge-shaped cracks in $1 \mathrm{D}$ hexagonal piezoelectric QCs bimaterial.

\section{Problem Description and Basic Equations}

The physical model considered in this section is shown in Figure 1. An interfacial crack of length $l$ and a wedge-shaped crack whose angle is $2 \alpha$ lie along a part of the interface between two 1D hexagonal piezoelectric QCs materials. We suppose that the interface is perfectly bonded along $x$-axis when $x \geq 0$. A screw dislocation of Burgers vector $\boldsymbol{b}=$ $\left(b_{3}, b_{\perp}, b_{\phi}\right)^{\mathrm{T}}$ is located at the point $z_{0}(r, \theta)$ in material 1 and the dislocation line is perpendicular to the $z$-plane. The crack surfaces are considered traction-free and no electric charge.

The problem considered is an antiplane elastic deformation problem. The nonzero field quantities $\left(\sigma_{z \beta}, \varepsilon_{z \beta}, u_{z}\right)$, $\left(H_{z \beta}, \omega_{z \beta}, w_{z}\right)$ and $\left(D_{\beta}, E_{\beta}, \phi\right), \beta=1,2$, should satisfy the following basic equations [12]:

constitutive equations

$$
\begin{gathered}
\sigma_{z \beta}=2 C_{44} \varepsilon_{z \beta}+R_{3} \omega_{z \beta}-e_{15} E_{\beta} \\
H_{z \beta}=2 R_{3} \varepsilon_{z \beta}+K_{2} \omega_{z \beta}-d_{15} E_{\beta} \\
D_{\beta}=2 e_{15} \varepsilon_{z \beta}+d_{15} \omega_{z \beta}+\lambda_{11} E_{\beta}
\end{gathered}
$$

geometry equations

$$
\begin{aligned}
\varepsilon_{z \beta} & =\frac{1}{2} u_{z, \beta}, \\
\omega_{z \beta} & =w_{z, \beta}, \\
E_{\beta} & =-\phi_{, \beta}
\end{aligned}
$$

equilibrium equations (if the body force is neglected)

$$
\begin{gathered}
\sigma_{z \beta, \beta}=0, \\
H_{z \beta, \beta}=0, \\
D_{\beta, \beta}=0
\end{gathered}
$$

where a comma represents partial differentiation and the repeated indices denote summation; $\sigma_{z \beta}, \varepsilon_{z \beta}$, and $u_{z}$ are the stresses, strains, and displacement of the phonon field, respectively, with $\beta=x, y ; H_{z \beta}, \omega_{z \beta}$, and $w_{z}$ are the stresses, strains, and displacement of the phason field, respectively; $D_{\beta}, E_{\beta}$, and $\phi$ represent the electric displacements, electric fields and electric potential, respectively; $C_{44}, K_{2}$, and $R_{3}$ denote the phonon elastic, phason elastic, and phononphason coupling constants, respectively; $e_{15}, d_{15}$ are the piezoelectric constants in the phonon field and phason field, respectively; $\lambda_{11}$ denotes the dielectric permittivity.

Substituting (1) and (2) into (3), the governing equations are obtained as follows:

$$
\boldsymbol{B} \nabla^{2} \boldsymbol{u}=0
$$

where $\boldsymbol{u}=\left(u_{z}, w_{z}, \phi\right)^{\mathrm{T}}, \nabla^{2}=\partial^{2} / \partial x^{2}+\partial^{2} / \partial y^{2}$ is the twodimensional Laplace operator and

$$
\boldsymbol{B}=\left(\begin{array}{ccc}
C_{44} & R_{3} & e_{15} \\
R_{3} & K_{2} & d_{15} \\
e_{15} & d_{15} & -\lambda_{11}
\end{array}\right)
$$

is the material constants matrix.

By using the properties of analytic function, in view of (4), one obtains

$$
\boldsymbol{u}=\operatorname{Im} \boldsymbol{f}(z)
$$

where Im represents the imaginary part of an analytic function.

Introducing the generalized strains $Z_{x}=\left[\varepsilon_{z x}, \omega_{z x},-E_{x}\right]^{\mathrm{T}}$, $Z_{y}=\left[\varepsilon_{z y}, \omega_{z y},-E_{y}\right]^{\mathrm{T}}$ and the generalized stresses $\boldsymbol{\Sigma}_{x}=$ $\left[\sigma_{z x}, H_{z x}, D_{x}\right]^{\mathrm{T}}, \Sigma_{y}=\left[\sigma_{z y}, H_{z y}, D_{y}\right]^{\mathrm{T}}$, we have the following relationships:

$$
\begin{aligned}
& \mathbf{Z}_{y}+i \mathbf{Z}_{x}=\boldsymbol{f}^{\prime}(z) \\
& \boldsymbol{\Sigma}_{y}+i \boldsymbol{\Sigma}_{x}=\boldsymbol{B} \boldsymbol{f}^{\prime}(z)
\end{aligned}
$$

where the superscript prime denotes the derivative with respect to the argument.

By (7), the resultant force and the normal component of the electric displacement along any arc $A B$ can be represented as

$$
\boldsymbol{T}=\int_{A}^{B} \boldsymbol{\Sigma}_{x} d y-\boldsymbol{\Sigma}_{y} d x=\boldsymbol{B} \operatorname{Re}[\boldsymbol{f}(z)]_{A}^{B}
$$

where Re indicates the real part of an analytical function.

The boundary conditions of the present problem can be expressed as follows:

$$
\begin{aligned}
\int_{l} d \boldsymbol{u}^{(1)} & =\boldsymbol{b}, \\
\int_{l} \Sigma_{y}^{(1)} d_{x}-\Sigma_{x}^{(1)} d_{y} & =0 \\
\boldsymbol{u}^{(1)}\left(x, 0^{+}\right) & =\boldsymbol{u}^{(2)}\left(x, 0^{-}\right), \\
\boldsymbol{T}^{(1)}\left(x, 0^{+}\right) & =\boldsymbol{T}^{(2)}\left(x, 0^{-}\right),
\end{aligned}
$$

$$
0 \leq x \leq \infty
$$




$$
\begin{aligned}
\boldsymbol{T}^{(1)}(x, 0) & =\boldsymbol{T}^{(2)}(x, 0)=0, \quad-l \leq x \leq 0 \\
\Sigma_{x}^{(1)} n_{x}^{(1)}+\Sigma_{y}^{(1)} n_{y}^{(1)}=0, & y=-(x+l) \tan \alpha \\
\Sigma_{x}^{(2)} n_{x}^{(2)}+\Sigma_{y}^{(2)} n_{y}^{(2)} & =0, \quad y=(x+l) \tan \alpha
\end{aligned}
$$

in which all quantities with superscripts $j(j=1,2)$ represent material 1 and material 2, respectively. The following main task is to determine the unknown functions $f^{(j)}(z)$ satisfying the boundary conditions.

\section{Solution to the Problem}

Introduce a conformal mapping function as [15]

$$
\begin{aligned}
& \zeta=\sqrt{(z+l)^{\delta}-l^{\delta}}, \\
& \delta=\frac{\pi}{\pi-\alpha},
\end{aligned}
$$

$$
1 \leq \delta \leq 2, \zeta=\xi+i \eta
$$

which transforms the infinite plane which consists of material 1 and material 2 in the $z$-plane into the upper and lower quarter planes in the $\zeta$-plane and the point $z_{0}$ in the $z$-plane into the point $\zeta_{0}=\sqrt{\left(z_{0}+l\right)^{\delta}-l^{\delta}}$ in the $\zeta$-plane.

By (10), (11), and (12), we have

$$
\begin{aligned}
& u^{(1)}\left(\xi, 0^{+}\right)=u^{(2)}\left(\xi, 0^{-}\right), \\
& T^{(1)}\left(\xi, 0^{+}\right)=\boldsymbol{T}^{(2)}\left(\xi, 0^{-}\right), \\
& \boldsymbol{T}^{(1)}(0, \eta)=0, \quad \eta \geq 0 \\
& \boldsymbol{T}^{(2)}(0, \eta)=0, \quad \eta \leq 0
\end{aligned}
$$

Assume that the unknown functions $f^{(j)}$ in the $\zeta$-plane can be expressed as

$$
\boldsymbol{f}^{(j)}(\zeta)=\boldsymbol{f}_{b}^{(j)}(\zeta)+\boldsymbol{f}_{c}^{(j)}(\zeta)
$$

where $\boldsymbol{f}_{b}{ }^{(j)}(\zeta)$ represent the functions of a screw dislocation in an infinite bimaterial, while $\boldsymbol{f}_{c}^{(j)}(\zeta)$ are the functions corresponding to the perturbed fields due to the free boundary $\zeta=0$.

We first consider a screw dislocation in a infinite 1D hexagonal piezoelectric QCs bimaterial. In this case, the functions $f_{b}{ }^{(j)}(\zeta)$, which satisfy the conditions (9) and (14), are assumed as

$$
\begin{aligned}
& \boldsymbol{f}_{b}^{(1)}(\zeta)=\boldsymbol{f}_{m}^{(1)}(\zeta)+\boldsymbol{f}_{p}^{(1)}(\zeta), \\
& \boldsymbol{f}_{b}^{(2)}(\zeta)=\boldsymbol{f}_{p}^{(2)}(\zeta)
\end{aligned}
$$

where $\boldsymbol{f}_{m}^{(1)}(\zeta)$ is connected to the unperturbed field and it is analytic apart from $\zeta_{0}$ point in the complex plane and $f_{p}^{(j)}(\zeta)$ is related to the perturbed fields in the material $j$.
By $(9), \boldsymbol{f}_{m}^{(1)}(\zeta)$ is given as

$$
\boldsymbol{f}_{m}^{(1)}(\zeta)=\frac{1}{2 \pi} \boldsymbol{b} \ln \left(\zeta-\zeta_{0}\right)
$$

Inserting (6) and (8) into (14), we have

$$
\begin{aligned}
\operatorname{Im}\left[\boldsymbol{f}_{b}{ }^{(1)}(\zeta)\right] & =\operatorname{Im}\left[\boldsymbol{f}_{b}{ }^{(2)}(\zeta)\right], \\
\boldsymbol{B}^{(1)} \operatorname{Re}\left[\boldsymbol{f}_{b}{ }^{(1)}(\zeta)\right] & =\boldsymbol{B}^{(2)} \operatorname{Re}\left[\boldsymbol{f}_{b}{ }^{(2)}(\zeta)\right]
\end{aligned}
$$

Using (17), (18), and (19), analytical extension techniques [16], and noting $\bar{\zeta}=\zeta$ holds along the real axis, we have

$$
\begin{aligned}
& \boldsymbol{f}_{b}{ }^{(1)}(\zeta)=\frac{1}{2 \pi}\left[\boldsymbol{I} \ln \left(\zeta-\zeta_{0}\right)+\boldsymbol{\Pi}_{12} \ln \left(\zeta-\bar{\zeta}_{0}\right)\right] \boldsymbol{b} \\
& \boldsymbol{f}_{b}{ }^{(2)}(\zeta)=\frac{1}{2 \pi}\left(\boldsymbol{I}-\boldsymbol{\Pi}_{12}\right) \boldsymbol{b} \ln \left(\zeta-\zeta_{0}\right)
\end{aligned}
$$

where the overbar is the conjugate of a complex function, $\boldsymbol{I}$ is a $3 \times 3$ unit matrix, and matrix $\Pi_{12}$ is

$$
\boldsymbol{\Pi}_{12}=\left(\boldsymbol{B}^{(2)}+\boldsymbol{B}^{(1)}\right)^{-1}\left(\boldsymbol{B}^{(2)}-\boldsymbol{B}^{(1)}\right)
$$

By (8), (15), and (16), noting $\bar{\zeta}=-\zeta$ holds along the imaginary axis, one can obtain

$$
\begin{aligned}
& \boldsymbol{f}_{c}^{(1)}(\zeta)=-\frac{1}{2 \pi}\left[\boldsymbol{I} \ln \left(\zeta+\bar{\zeta}_{0}^{-1}\right)+\boldsymbol{\Pi}_{12} \ln \left(\zeta+\zeta_{0}\right)\right] \boldsymbol{b} \\
& \boldsymbol{f}_{c}^{(2)}(\zeta)=-\frac{1}{2 \pi}\left(\boldsymbol{I}-\boldsymbol{\Pi}_{12}\right) \ln \left(\zeta+\bar{\zeta}_{0}\right) \boldsymbol{b}
\end{aligned}
$$

Substituting (20) and (22) into (16) and by (13), the functions $f^{(j)}(z)$ in the $z$-plane are obtained as

$$
\begin{aligned}
& \boldsymbol{f}^{(1)}(z)=\frac{1}{2 \pi}\left[\boldsymbol{I} \ln \frac{(z+l)^{\delta}-\left(z_{0}+l\right)^{\delta}}{(z+l)^{\delta}-\left(\bar{z}_{0}+l\right)^{\delta}}\right. \\
& \left.+\left(\boldsymbol{I}+\boldsymbol{\Pi}_{12}\right) \ln \frac{\zeta-\bar{\zeta}_{0}}{\zeta+\zeta_{0}}\right] \boldsymbol{b} \\
& \boldsymbol{f}^{(2)}(z)=\frac{1}{2 \pi}\left[\left(\boldsymbol{I}-\boldsymbol{\Pi}_{12}\right) \ln \frac{\zeta-\zeta_{0}}{\zeta+\bar{\zeta}_{0}}\right] \boldsymbol{b}
\end{aligned}
$$

With (23), the elastic and electric fields can be derived in terms of (6)-(7). For the generalized stress fields, one has

$$
\begin{aligned}
& \Sigma_{y}^{(1)}+i \Sigma_{x}^{(1)}=\frac{\delta(z+l)^{\delta-1}}{2 \pi}\left\{\boldsymbol { B } ^ { ( 1 ) } \left[\frac{1}{(z+l)^{\delta}-\left(z_{0}+l\right)^{\delta}}\right.\right. \\
& \left.-\frac{1}{(z+l)^{\delta}-\left(\bar{z}_{0}+l\right)^{\delta}}\right]+\boldsymbol{B}^{e f f}\left(\frac{1}{\zeta-\bar{\zeta}_{0}}-\frac{1}{\zeta+\zeta_{0}}\right) \\
& \left.\cdot \frac{1}{2 \zeta}\right\} \boldsymbol{b} \\
& \Sigma_{y}^{(2)}+i \Sigma_{x}^{(2)}=\frac{\delta(z+l)^{\delta-1}}{2 \pi} \boldsymbol{B}^{e f f} \frac{1}{2 \zeta}\left(\frac{1}{\zeta-\zeta_{0}}-\frac{1}{\zeta+\bar{\zeta}_{0}}\right) \\
& \cdot \boldsymbol{b}
\end{aligned}
$$

with $\boldsymbol{B}^{\text {eff }}=2 \boldsymbol{B}^{(2)}\left(\boldsymbol{B}^{(2)}+\boldsymbol{B}^{(1)}\right)^{-1} \boldsymbol{B}^{(1)}=2 \boldsymbol{B}^{(1)}\left(\boldsymbol{B}^{(2)}+\boldsymbol{B}^{(1)}\right)^{-1} \boldsymbol{B}^{(2)}$. 


\section{Stress Intensity Factors and Image Force on the Dislocation}

The phonon field stresses, phason field stresses, and electric displacements intensity factors at the crack tip are defined as

$$
\boldsymbol{K}=\left\{\begin{array}{l}
\boldsymbol{K}_{\sigma} \\
\boldsymbol{K}_{H} \\
\boldsymbol{K}_{D}
\end{array}\right\}=\lim _{z \longrightarrow 0} \sqrt{2 \pi z} \boldsymbol{\Sigma}_{y}^{(j)}, \quad j=1,2
$$

Substituting (24) into (25) will yield

$$
\boldsymbol{K}=-\frac{1}{2} \sqrt{\frac{\delta l^{\delta-1}}{2 \pi}} \boldsymbol{B}^{\text {eff }}\left(\frac{1}{\zeta_{0}}+\frac{1}{\bar{\zeta}_{0}}\right) \boldsymbol{b}
$$

According to the generalized Peach-Koehler formula [14], the image force is expressed as

$$
\boldsymbol{F}_{x}-i \boldsymbol{F}_{y}=\boldsymbol{b}^{\mathrm{T}}\left[\boldsymbol{\Sigma}_{y p}^{(1)}\left(z_{0}\right)+i \boldsymbol{\Sigma}_{x p}^{(1)}\left(z_{0}\right)\right]
$$

in which $\Sigma_{k p}^{(1)}\left(z_{0}\right)(k=x, y)$ are the perturbation stresses of the phonon fields, phason fields, and the perturbed electric displacements at the dislocation point $z_{0}$. With (7), (24), and (27), we have

$$
\begin{aligned}
& \boldsymbol{F}_{x}-i \boldsymbol{F}_{y}=\frac{\delta(z+l)^{\delta-1}}{2 \pi} \\
& \cdot \boldsymbol{b}^{\mathrm{T}}\left\{\boldsymbol{B}^{(1)}\left[\frac{\delta-1}{2 \delta\left(z_{0}+l\right)^{\delta}}-\frac{1}{\left(z_{0}+l\right)^{\delta}-\left(\bar{z}_{0}+l\right)^{\delta}}\right]\right. \\
&\left.+\boldsymbol{B}^{e f f}\left(\frac{1}{\zeta_{0}-\bar{\zeta}_{0}}-\frac{1}{2 \zeta_{0}}\right) \frac{1}{2 \zeta_{0}}\right\} \boldsymbol{b}
\end{aligned}
$$

The analytical solutions of some special cases can be derived from the present results. Discussions in detail are given as follows:

(1) If the length of the interfacial crack $l$ tends to zero, (24) reduces to

$$
\begin{gathered}
\Sigma_{y}^{(1)}+i \Sigma_{x}^{(1)}=\frac{\delta}{4 \pi z^{(1-\delta / 2)}}\left[\boldsymbol { B } ^ { ( 1 ) } \left(\frac{1}{\sqrt{z^{\delta}}-\sqrt{z_{0}^{\delta}}}\right.\right. \\
\left.-\frac{1}{\sqrt{z^{\delta}}+\sqrt{\bar{z}_{0}^{\delta}}}\right)+\boldsymbol{B}^{(1)} \Pi_{12}\left(\frac{1}{\sqrt{z^{\delta}}-\sqrt{\bar{z}_{0}^{\delta}}}\right. \\
\left.\left.-\frac{1}{\sqrt{z^{\delta}}+\sqrt{z_{0}^{\delta}}}\right)\right] \boldsymbol{b}
\end{gathered}
$$

Equation (29) represents the case of the interaction of a screw dislocation with a wedge-shaped crack. If $\boldsymbol{B}^{(1)}=\boldsymbol{B}^{(2)}$, the result is in excellent agreement with the result in [17].
(2) When $\beta=0$, (24) degenerates into

$$
\begin{aligned}
& \Sigma_{y}^{(1)}+i \Sigma_{x}^{(1)}=\frac{1}{2 \pi}\left[\boldsymbol{B}^{(1)}\left(\frac{1}{z-z_{0}}-\frac{1}{z-\bar{z}_{0}}\right)\right. \\
& \left.+\boldsymbol{B}^{e f f}\left(\frac{1}{\sqrt{z}-\sqrt{\bar{z}_{0}}}-\frac{1}{\sqrt{z}+\sqrt{z_{0}}}\right) \frac{1}{2 \sqrt{z}}\right] \boldsymbol{b}
\end{aligned}
$$

Equation (30) gives the case of a dislocation interacting with interface and a semi-infinite interfacial crack.

(3) When $\beta=\pi / 2$, (24) becomes

$$
\begin{aligned}
& \Sigma_{y}^{(1)}+i \Sigma_{x}^{(1)}=\frac{1}{2 \pi}\left[\boldsymbol { B } ^ { ( 1 ) } \left(\frac{1}{z-z_{0}}+\frac{1}{z-z_{0}+2 l}\right.\right. \\
& \left.-\frac{1}{z-\bar{z}_{0}}-\frac{1}{z-\bar{z}_{0}+2 l}\right) \\
& +\boldsymbol{B}^{e f f}\left(\frac{1}{\sqrt{z(z+2 l)}-\sqrt{\bar{z}_{0}\left(\bar{z}_{0}+2 l\right)}}\right. \\
& \left.\left.-\frac{1}{\sqrt{z(z+2 l)}+\sqrt{z_{0}\left(z_{0}+2 l\right)}}\right) \frac{z+l}{\sqrt{z(z+2 l)}}\right] \boldsymbol{b}
\end{aligned}
$$

Equation (31) represents the result of the interaction of a dislocation with interface and a finite interfacial crack.

\section{Numerical Results}

In this section, the selected numerical results are presented to analyze the fracture mechanic behavior of the 1D hexagonal piezoelectric QCs bimaterial. The material properties of the bimaterial are listed in Table 1 [16, 18-20].

The coupling constant $R$ has great influence on the mechanical behaviors of QCs, and it has not been measured yet. In the computation, the coupling constant of material 2 is assumed as $R_{3}^{(2)}=0.8846 \mathrm{GPa}$ [21].

Other parameters are selected as follows:

$$
\begin{aligned}
& b_{3}=1.6 \times 10^{-9} \mathrm{~m}, \\
& b_{\perp}=10.7 \times 10^{-9} \mathrm{~m}, \\
& b_{\phi}=1 \times 10^{-9} \mathrm{~V} .
\end{aligned}
$$

Figures 2 and 3 show the variations of the normalized phonon and phason stresses intensity factors with the angular position $\theta$ of the dislocation for different values of $R_{3}^{(1)}$ at $r / l=0.1, \alpha=\pi / 6$. It is found that the normalized phonon and phason stresses intensity factors are always negative, indicating that screw dislocation can reduce the shielding effect.

Figures 4 and 5 depict the normalized force on the dislocation versus $\theta$ for different values of the wedge angle $\alpha$ 
TABLE 1: Material properties of two 1D hexagonal piezoelectric QCs.

\begin{tabular}{lccccc}
\hline & $C_{44}(G P a)$ & $K_{2}(G P a)$ & $e_{15}\left(\mathrm{C} \cdot \mathrm{m}^{-2}\right)$ & $d_{15}\left(\mathrm{C} \cdot \mathrm{m}^{-2}\right)$ & $\lambda_{11}\left(10^{-9} \mathrm{C}^{2} \mathrm{~N}^{-1} \mathrm{~m}^{-2}\right)$ \\
\hline Material 1 & 50 & 0.3 & -0.318 & -0.16 & 0.0826 \\
Material 2 & 70.19 & 24 & 11.6 & 1.16 & 5 \\
\hline
\end{tabular}

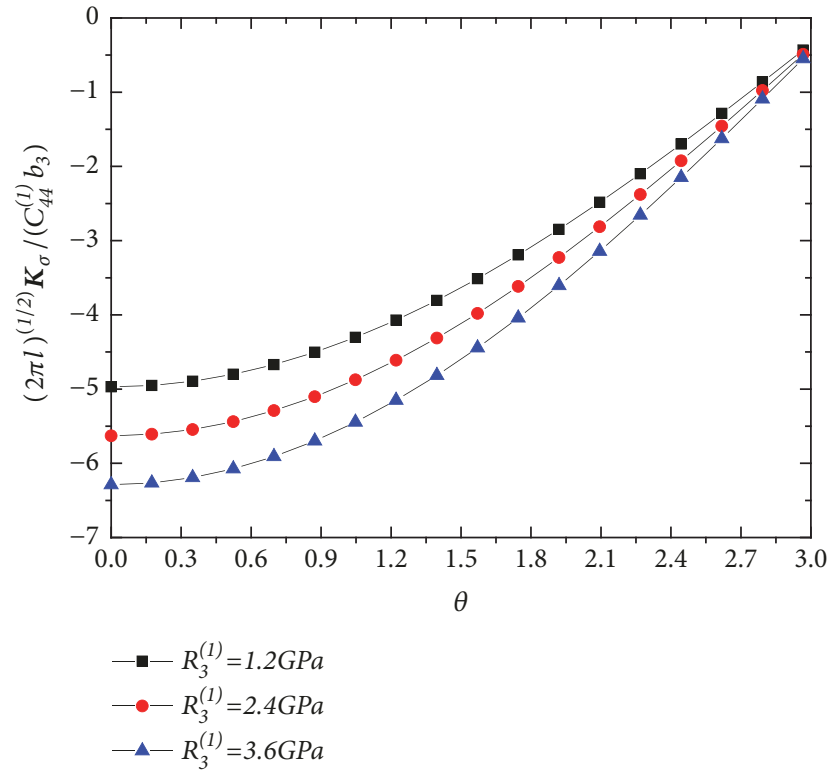

FIgURE 2: Normalized phonon stresses intensity factor $(2 \pi l)^{1 / 2} \boldsymbol{K}_{\sigma} /\left(C_{44}^{(1)} b_{3}\right)$ versus $\theta$.

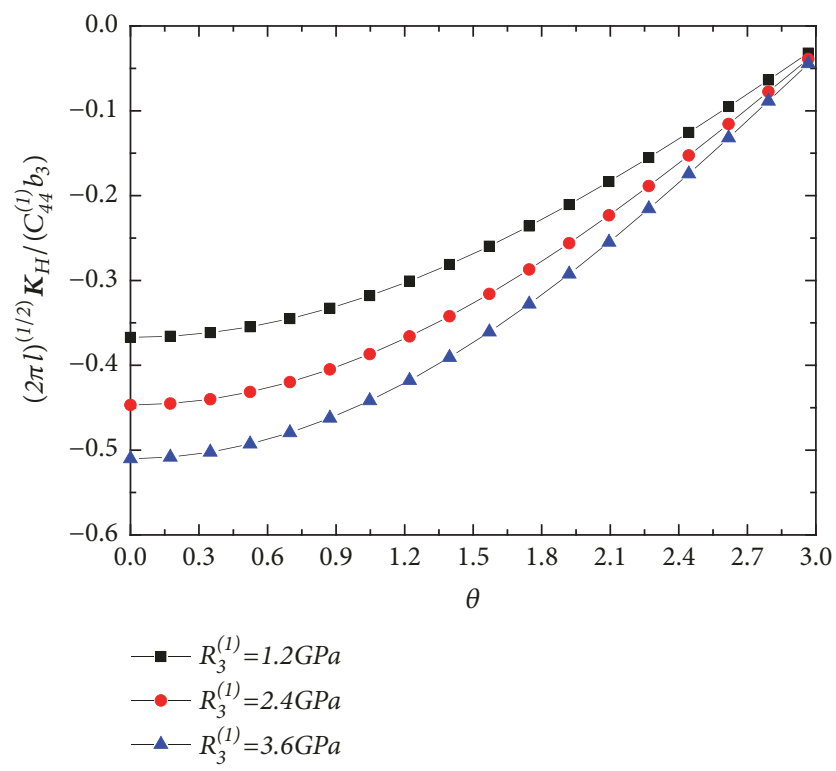

FIgURE 3: Normalized phason stresses intensity factor $(2 \pi l)^{1 / 2} \boldsymbol{K}_{H} /$ $\left(C_{44}^{(1)} b_{3}\right)$ versus $\theta$.

at $r / 1=0.5$. It can be seen from Figure 4 that the normalized image force along $x$-axis is always negative and the absolute value decreases with the increase of the wedge angle $\alpha$ when $0<\theta<\pi / 2$ and increases with the increase of $\alpha$ when

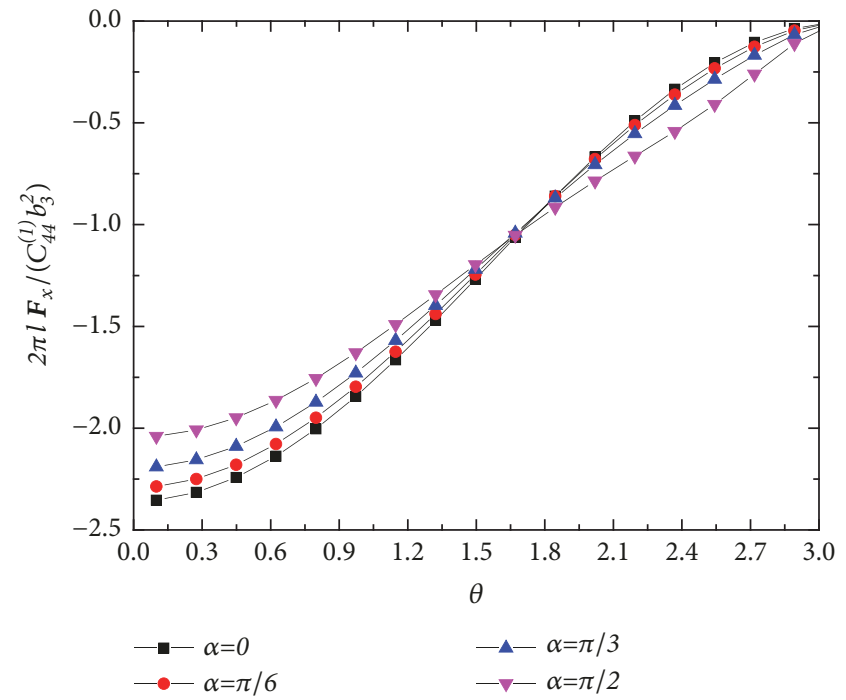

FIGURE 4: Normalized image force $2 \pi l F_{x} /\left(C_{44}^{(1)} b_{3}^{2}\right)$ versus $\theta$.

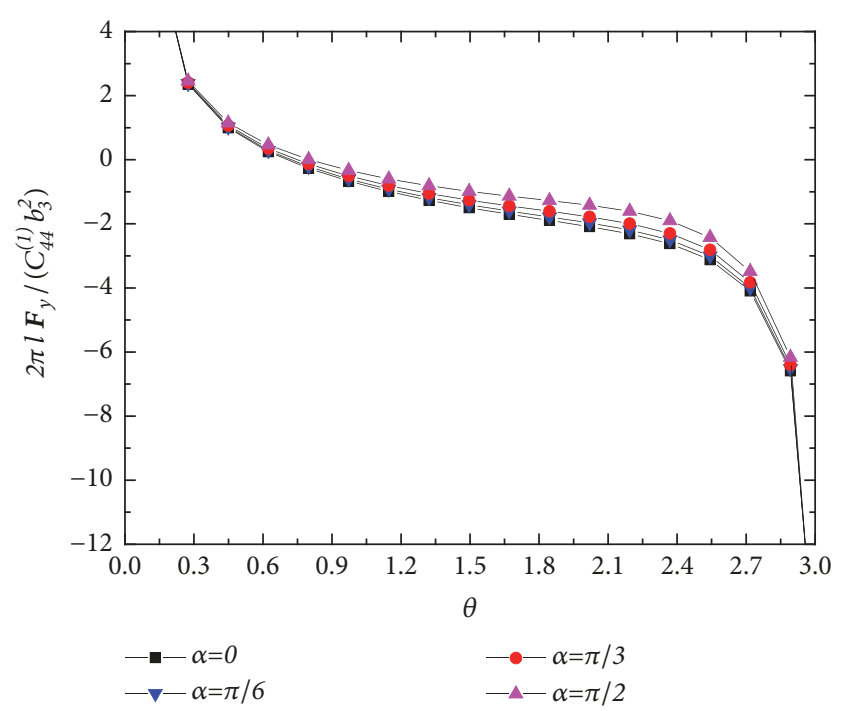

FIGURE 5: Normalized image force $2 \pi l \boldsymbol{F}_{y} /\left(C_{44}^{(1)} b_{3}^{2}\right)$ versus $\theta$.

$\pi / 2<\theta<\pi$. Figure 5 shows that the wedge angle $\alpha$ has no obvious effect on the normalized image force along $y$-axis.

Figures 6,7 , and 8 show the distribution of the phonon stresses, phason stresses, and electric displacements with $r=1, \theta=\pi / 6$, and $r / l=0.5$. It can be seen that the phonon stresses, phason stresses, and electric displacements are singular at the crack tip $z=0$ and dislocation point $z=z_{0}$. $\sigma_{z y}, H_{z y}$, and $D_{y}$ are continuous at the interface $y=0$, while $\sigma_{z x}, H_{z x}$, and $D_{x}$ are discontinuous at the interface $y=0$. 


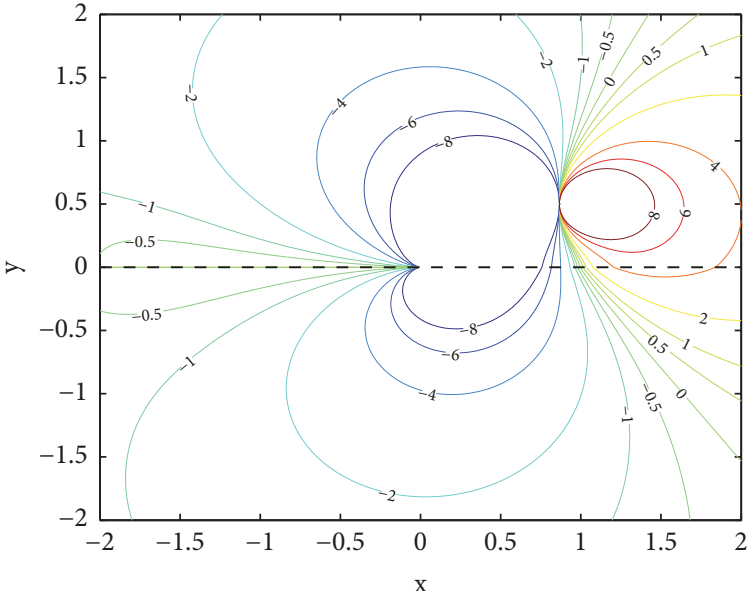

(a) $10^{-10}\left(2 \pi \sigma_{z y} / b_{3}\right)$

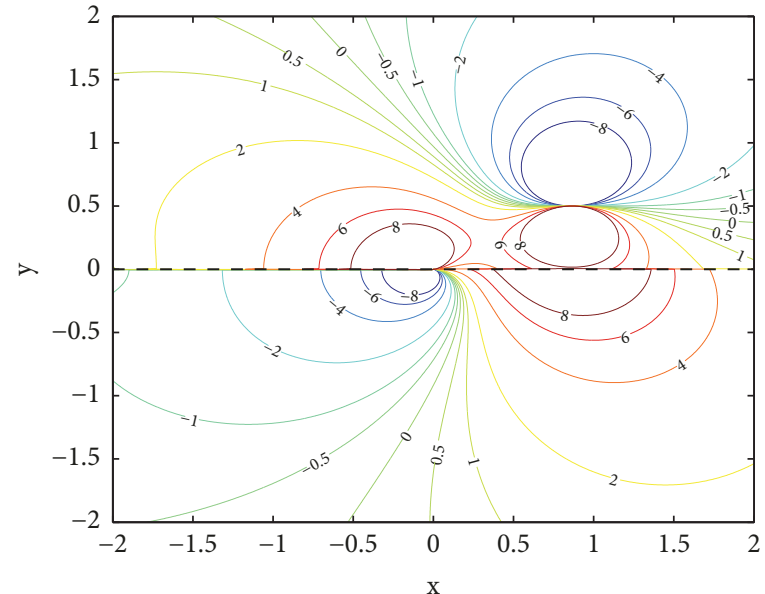

(b) $10^{-10}\left(2 \pi \sigma_{z x} / b_{3}\right)$

Figure 6: Contours of the phonon stresses.

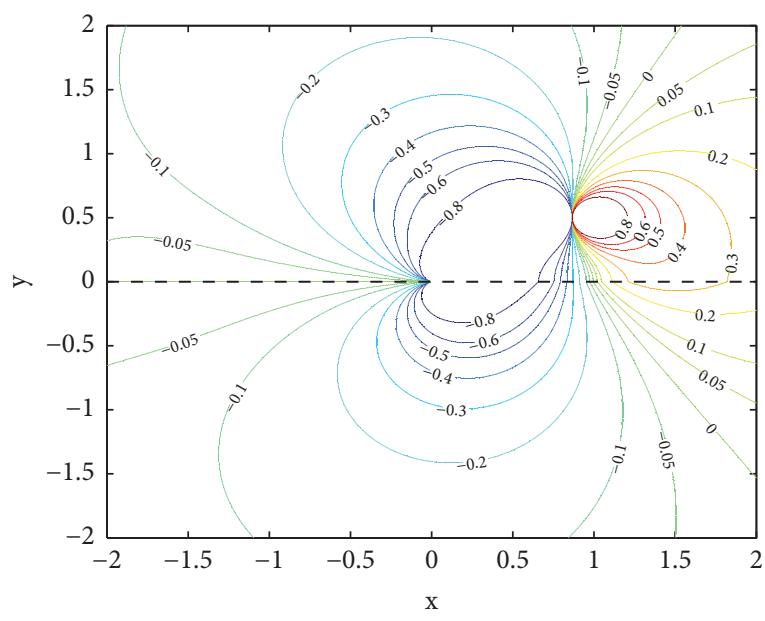

(a) $10^{-10}\left(2 \pi H_{z y} / b_{3}\right)$

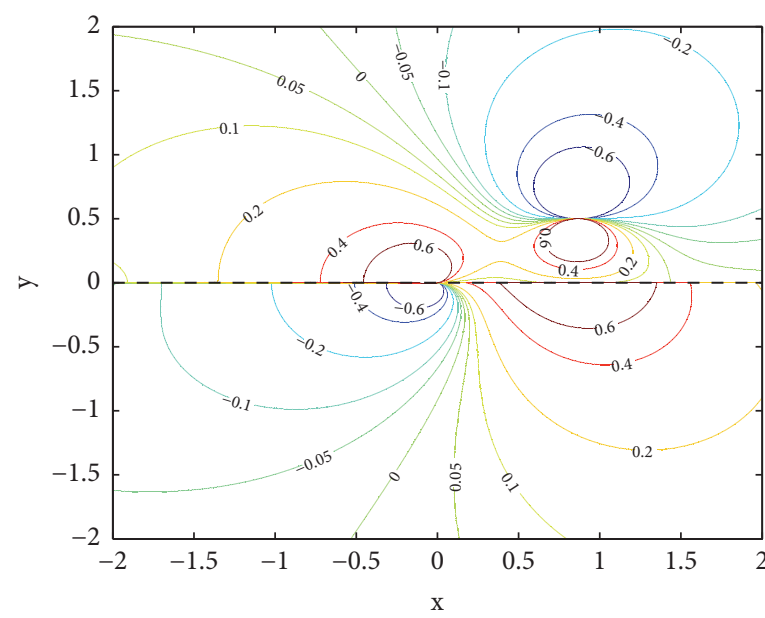

(b) $10^{-10}\left(2 \pi H_{z x} / b_{3}\right)$

FIGURE 7: Contours of the phason stresses.

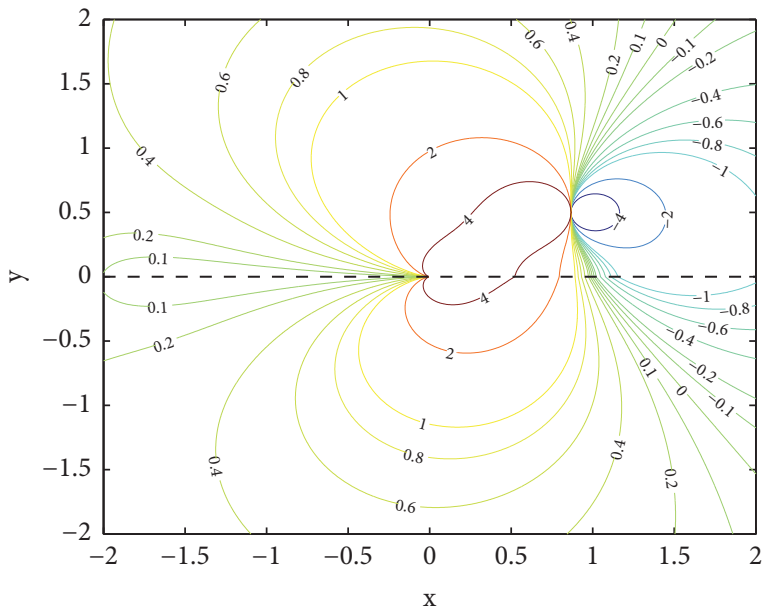

(a) $2 \pi D_{y} / b_{3}$

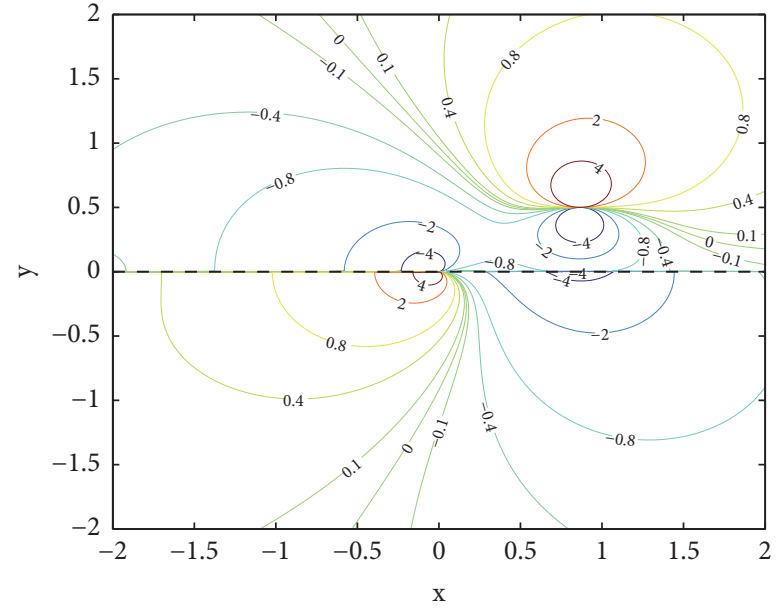

(b) $2 \pi D_{x} / b_{3}$

FIGURE 8: Contours of the electric displacements. 


\section{Conclusions}

The wedge-shaped cracks interacting with a screw dislocation in 1D hexagonal piezoelectric QCs bimaterial are presented in this paper. The analytic expressions of the electrical field, phason, and phonon field have been derived. The solutions obtained in present paper can be generalized to other QCs bimaterial problem. These results provide the theoretical basis for the application of the QCs material.

\section{Data Availability}

The data used to support the findings of this study are included within the article.

\section{Conflicts of Interest}

The authors declare that there are no conflicts of interest regarding the publication of this paper.

\section{Acknowledgments}

This work is supported by the National Natural Science Foundation of China (Grants nos. 11462020 and 11361039) and the Inner Mongolia Natural Science Foundation (Grants nos. 2017MS0104, 2015MS0129, and NJZY18022), which are gratefully acknowledged.

\section{References}

[1] D. Shechtman, I. Blech, D. Gratias, and J. W. Cahn, "Metallic phase with long-range orientational order and no translational symmetry," Physical Review Letters, vol. 53, no. 20, pp. 1951-1953, 1984.

[2] C. Hu, R. Wang, and D. Ding, "Symmetry groups, physical property tensors, elasticity and dislocations in quasicrystals," Reports on Progress in Physics, vol. 63, no. 1, pp. 1-39, 2000.

[3] X.-Y. Li, "Elastic field in an infinite medium of one-dimensional hexagonal quasicrystal with a planar crack," International Journal of Solids and Structures, vol. 51, no. 6, pp. 1442-1455, 2014.

[4] T. Y. Fan and Y. F. Sun, "A moving screw dislocation in a onedimensional hexagonal quasicrystal," Acta Physica Sinica, vol. 8, no. 4, pp. 288-295, 1999.

[5] X. F. Li, Y. F. Sun, and T. Y. Fan, "Elastic field of a straight dislocation in one dimensional hexagonal quasicrystals," Journal of Beijing Institute of Technology, vol. 212, no. 1, pp. 66-71, 1999.

[6] E. Radi and P. M. Mariano, "Stationary straight cracks in quasicrystals," International Journal of Fracture, vol. 166, no. 1-2, pp. 105-120, 2010.

[7] Y. Gao, A. Ricoeur, and L. Zhang, "Plane problems of cubic quasicrystal media with an elliptic hole or a crack," Physics Letters A, vol. 375, no. 28-29, pp. 2775-2781, 2011.

[8] X. Wang and Z. Zhong, "Interaction between a semi-infinite crack and a straight dislocation in a decagonal quasicrystal," International Journal of Engineering Science, vol. 42, no. 5-6, pp. 521-538, 2004.

[9] G.-T. Liu, R.-P. Guo, and T.-Y. Fan, "On the interaction between dislocations and cracks in one-dimensional hexagonal quasicrystals," Chinese Physics, vol. 12, no. 10, pp. 1149-1155, 2003.
[10] L. H. Li and G. T. Liu, "Interaction of a dislocation with an elliptical hole in icosahedral quasicrystals," Philosophical Magazine Letters, vol. 93, no. 3, pp. 142-151, 2013.

[11] K. Rama Mohana Rao, P. Hemagiri Rao, and B. S. K. Chaitanya, "Piezoelectricity in quasicrystals: a group-theoretical study," Pramana-Journal of Physics, vol. 68, no. 3, pp. 481-487, 2007.

[12] G. Altay and M. Cengiz Dökmeci, "On the fundamental equations of piezoelasticity of quasicrystal media," International Journal of Solids and Structures, vol. 49, no. 23-24, pp. 3255$3262,2012$.

[13] J. Yu, J. Guo, and Y. Xing, "Complex variable method for an anti-plane elliptical cavity of one-dimensional hexagonal piezoelectric quasicrystals," Chinese Journal of Aeronautics, vol. 28, no. 4, pp. 1287-1295, 2015.

[14] J. Yang and X. Li, "Analytic solutions of problem about a circular hole with a straight crack in one-dimensional hexagonal quasicrystals with piezoelectric effects," Theoretical and Applied Fracture Mechanics, vol. 82, pp. 17-24, 2016.

[15] J.-X. Liu and X.-Q. Wang, "Interaction of a screw dislocation with a notch in a piezoelectric bi-material," Archive of Applied Mechanics, vol. 73, no. 8, pp. 553-560, 2004.

[16] Y. Juan and L. Xing, "The anti-plane shear problem of two symmetric cracks originating from an elliptical hole in 1D hexagonal piezoelectric QCS," Advanced Materials Research, vol. 936, pp. 127-135, 2014.

[17] L.-J. Jiang and G.-T. Liu, “The interaction between a screw dislocation and a wedge-shaped crack in one-dimensional hexagonal piezoelectric quasicrystals," Chinese Physics B, vol. 26, no. 4, pp. 245-251, 2017.

[18] J. Yu, J. Guo, E. Pan, and Y. Xing, "General solutions of plane problem in one-dimensional quasicrystal piezoelectric materials and its application on fracture mechanics," Applied Mathematics and Mechanics-English Edition, vol. 36, no. 6, pp. 793-814, 2015.

[19] J. Guo, Z. Zhang, and Y. Xing, "Antiplane analysis for an elliptical inclusion in $1 \mathrm{D}$ hexagonal piezoelectric quasicrystal composites," Philosophical Magazine, vol. 96, no. 4, pp. 349-369, 2016.

[20] C. Fan, Y. Li, G. Xu, and M. Zhao, "Fundamental solutions and analysis of three-dimensional cracks in one-dimensional hexagonal piezoelectric quasicrystals," Mechanics Research Communications, vol. 74, pp. 39-44, 2016.

[21] L. Zhang, D. Wu, W. Xu et al., "Green's functions of onedimensional quasicrystal bi-material with piezoelectric effect," Physics Letters A, vol. 380, no. 39, pp. 3222-3228, 2016. 


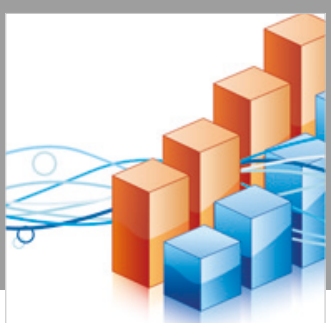

Advances in

Operations Research

\section{-n-m}
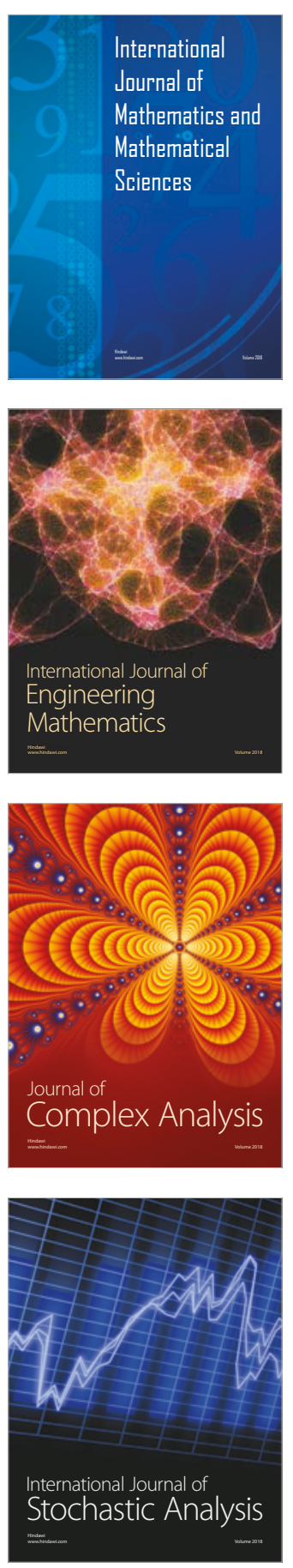
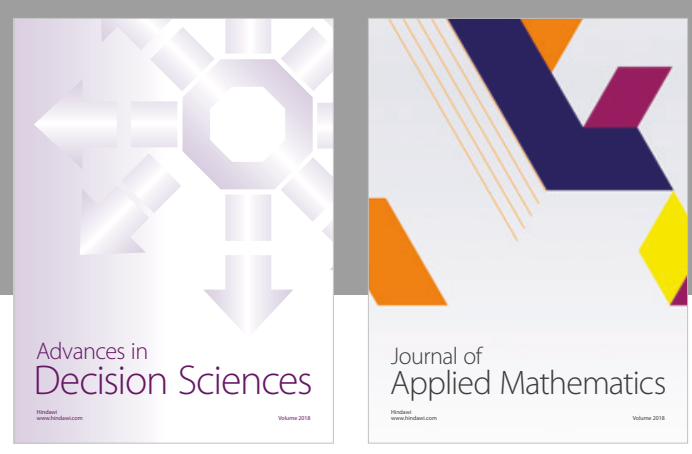

Journal of

Applied Mathematics
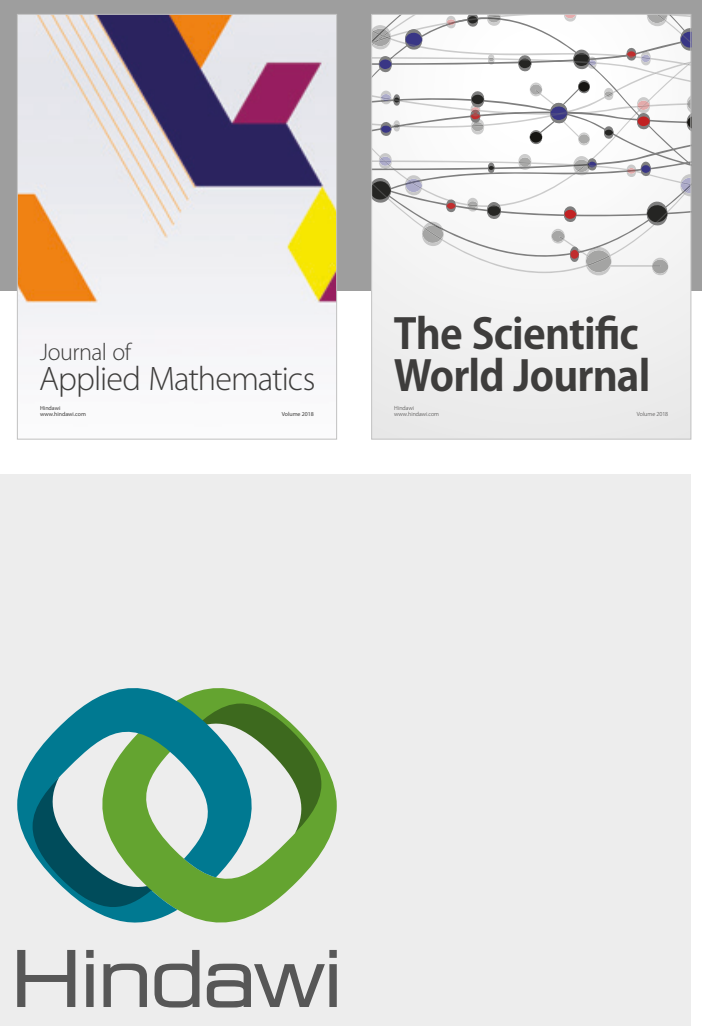

Submit your manuscripts at

www.hindawi.com

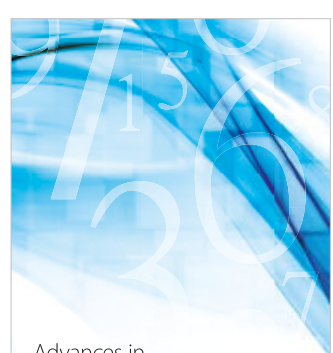

Advances in
Numerical Analysis
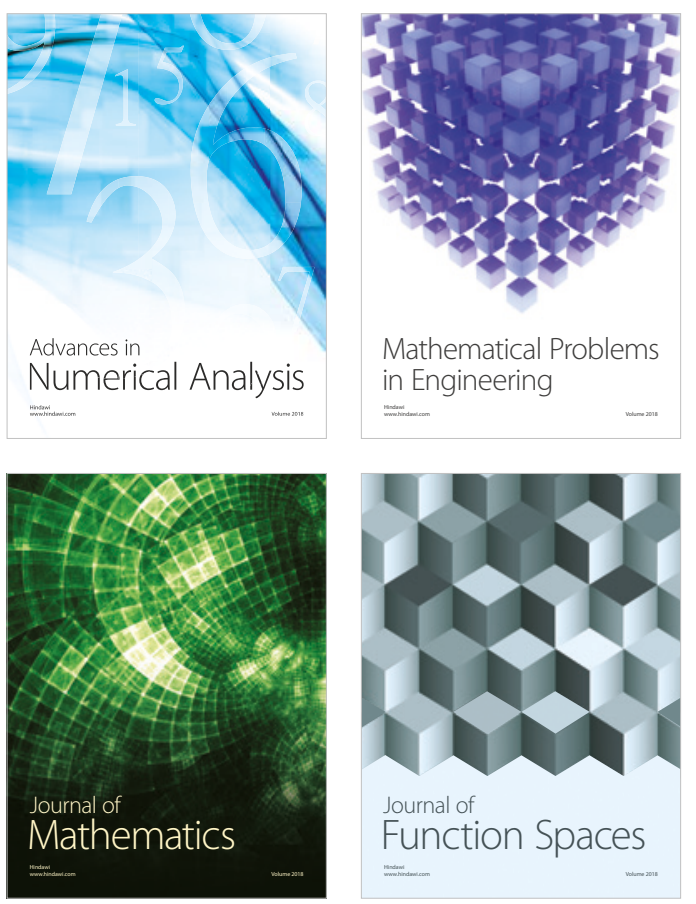

Mathematical Problems in Engineering

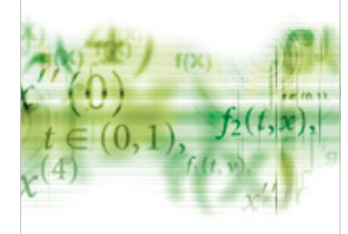

International Journal of

Differential Equations

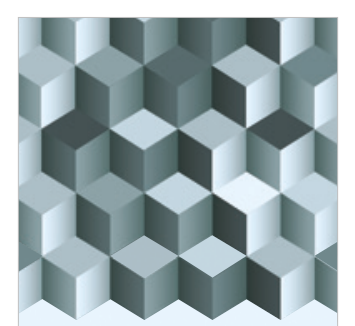

Journal of

Function Spaces

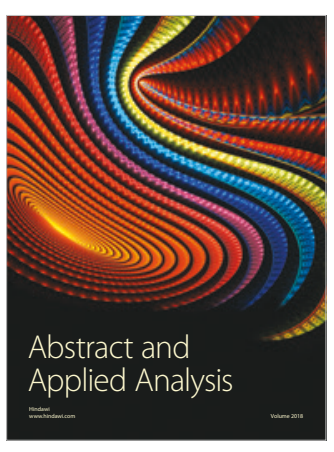

The Scientific

World Journal

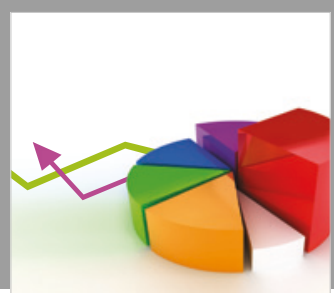

Journal of

Probability and Statistics
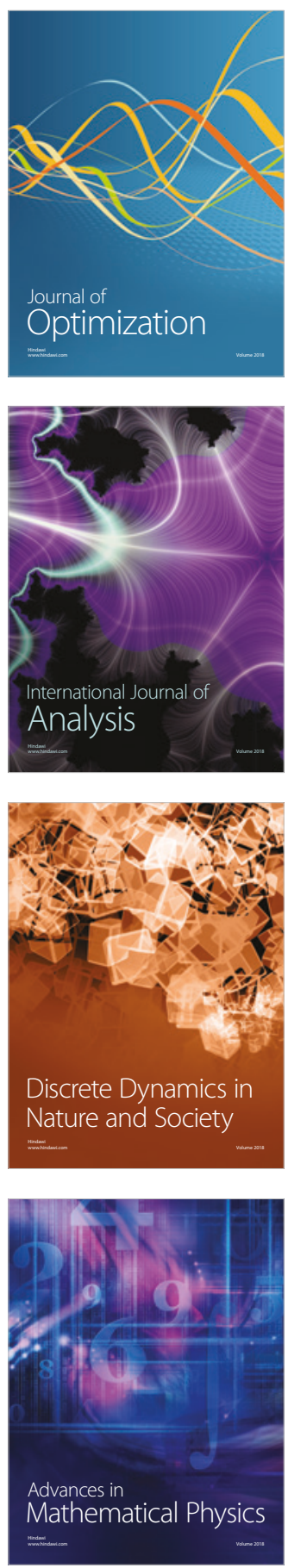